\title{
Ultrasonic manifestation of urethral polyp in a girl: a case report
}

\author{
Lin $\mathrm{Du}^{1}$, Yupeng $\mathrm{Zhu}^{2}$, Yan $\mathrm{Li}^{2}$, Ying $\mathrm{Sun}^{2}$ \\ ${ }^{1}$ Department of Developmental and Behavioral Pediatrics, ${ }^{2}$ Department of Abdominal Ultrasonography, The First Hospital of Jilin University, \\ Changchun 130021, China \\ Correspondence to: Ying Sun. Department of Ultrasonography, The First Hospital of Jilin University, No. 71 Xinmin Street, Chaoyang District, \\ Changchun 130021, China. Email: y_sun@jlu.edu.cn.
}

\begin{abstract}
Urethral polyp, a rare condition, occurs frequently in boys but seldom in young girls. In this report, we describe a case of a six-year-old female patient who experienced dysuria for eight days. Color ultrasound of the patient's urinary system revealed a solid nodule $14 \times 9 \mathrm{~mm}$ in size at the opening of the urethra in the trigonal area of the bladder. The nodule was connected to the urethra's posterior wall, and no blood flow signal could be detected. The primary diagnosis was urethral mass. A F7 ureteroscope was used as the cystourethroscope and was inserted into the bladder via the external opening of the urethra. Biopsy forceps were used to remove pathological lesions from the uplifted mucosa and submucosa. The postoperative pathological report was urethral polyp. When a child experiences intermittent urine retention, intermittent hematuria, and intermittent lower urinary tract symptoms, urethral polyp should be considered first.
\end{abstract}

Keywords: Urethral polyp; child; ultrasound; case report

Submitted Jan 14, 2020. Accepted for publication Feb 24, 2020.

doi: $10.21037 /$ tp.2020.03.05

View this article at: http://dx.doi.org/10.21037/tp.2020.03.05

\section{Introduction}

Urethral polyp, a rare condition, occurs frequently in boys but seldom in young girls. In boys, urinary polyps usually cause obstruction of the urethra (1); however, young girls typically experience no symptoms or only hematuria. This case report describes urethral polyp in a 6-yearold girl. Dysuria was the girl's first symptom, and she was first diagnosed with urethral polyp with ultrasound. Postoperative pathological examination showed the resected tissues to be inflammatory polyps. The characteristics of this case are unique compared with existing literature (2).

\section{Case presentation}

A 6-year-old female patient who had complained of dysuria for eight days was admitted to hospital after a urethral mass was detected by physical examination four days ago.

The characteristics of this case are as follows:

(I) A female child, who experienced dysuria for eight days, was found to have a urethral mass by physical examination four days ago.
(II) Eight days ago, the girl started having difficulty urinating without any obvious inducement. The symptom was not a cause of much concern for her parents. Four days ago, the symptom was still persisting without any relief, so the girl was sent to the outpatient department of our hospital. Color ultrasound of the girl's urinary system indicated a urethral mass. Through systematic physical examination, she was admitted to our hospital after being diagnosed with urethral mass. During the course of her condition, the girl encountered no discomfort in either side of the waist, no hematuria, no turbid urine, and no significant changes in appetite, sleep, or weight.

(III) Physical examination: the waist was symmetrical; there was no tenderness at the costal ridge point or costal lumbar point; and percussion pain in the double kidney area was negative. There was no tenderness in the bilateral ureteral region, and no swelling or tenderness in the suprapubic bladder region.

(IV) Auxiliary examination: Color ultrasound of the patient's urinary system (Figure 1) showed that the 


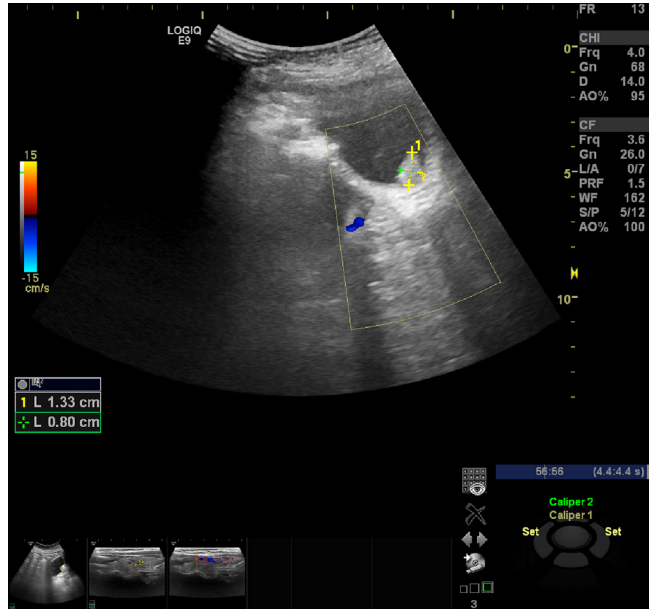

Figure 1 Ultrasonography showed a solid nodule at the opening of the urethra in the trigonal area of the bladder. The nodule was connected to the posterior wall of the urethra, and no blood flow signal could be detected.

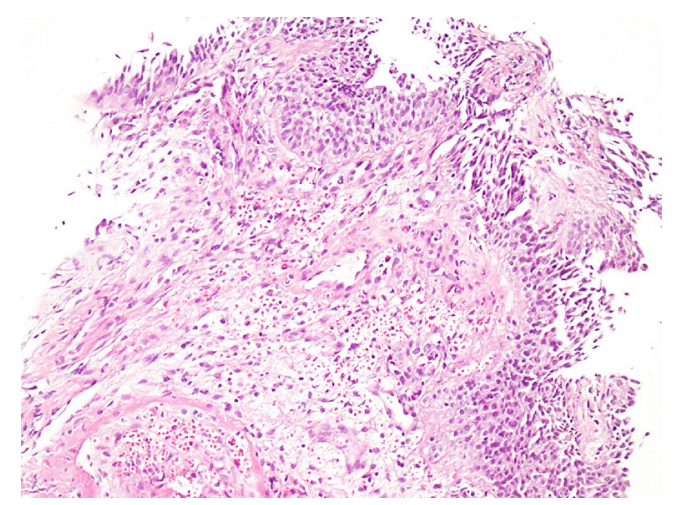

Figure 2 Biopsy histology (hematoxylin-eosin staining, $\times 200$ ) showing subepithelial infiltration of inflammatory cells.

left kidney was $79 \times 33 \mathrm{~mm}$ in size and the right kidney was $77 \times 35 \mathrm{~mm}$ in size. The size, shape, and structure of both kidneys were normal. The renal sinuses of both kidneys were not separated. The ureters were not dilated. A solid nodule $14 \times 9 \mathrm{~mm}$ in size was detected at the opening of the urethra in the trigonal area of the bladder. The nodule was connected to the posterior wall of the urethra, and no blood flow signal could be detected.

Pelvic multi row CT plain scan: the bladder was highly filled, and the bladder wall was not thick. No abnormal density shadow was found in the cavity. The primary diagnosis was urethral mass, and the chosen treatment was selective operation after improving the relevant medical examination.

Operation process: the lithotomy position was adopted. A F7 ureteroscope was used as the cystourethroscope and was inserted into the bladder through the external opening of the urethra. The bladder was filled with physiological saline and then observed. The ureteral openings on both sides were clearly visible, and the urine spray was clear. No other changes, such as cysts or valves, were found. The rest of the bladder appeared normal. A hill-like mucosal upheaval, with a length of $1.5 \mathrm{~cm}$ and a height of $0.5 \mathrm{~cm}$, was positioned at 5 to 7 o'clock from the middle part of the urethra's inner orifice. There was also a bulge of mucosa set in the direction of 9 to 10 o'clock in the urethra. The surface of the bulge was not smooth, but no vegetablelike swelling or other changes were found to exist. The texture of the lesions was soft when touched with the microscope body and biopsy forceps. Biopsy forceps were used to remove a number of small pathological lesions from the uplifted mucosa and submucosa at the 5 to 7 o'clock position. No tumor changes, such as leiomyoma, were found under the mucosa. The tumor tissue was resected and sent for histopathological examination after the operation. The pathological report was polyp of urethritis (Figure 2).

The patient's guardian gave his informed written consent for the case to be used in this study. The research protocol observed the tenets of the Declaration of Helsinki and was approved by the ethics committee of the First Hospital of Jilin University (No. 2017-428).

\section{Discussion}

Urethral polyp is a rare disease, rarely reported in literature. In children, urethral polyps mostly occur in boys and are only sporadically reported in girls. The study of the etiology and histology of urethral polyp has not yet been thoroughly investigated. In boys, some urethral polyps, usually fibroepithelial polyps, are considered to be congenital (3). Stephens considered that polyps in boys were hamartomas originating from the urethra and the prostate, showing the persistence of Muller's nodule. The stem of the polyp was found to be formed by the extension of the central nucleus and blood vessels of the epithelium and fibrous tissue of the genitourinary sinus (1). Ben-Meir et al. reported five cases of urinary polyp in young girls. The etiology of most of the polyps was speculated to be same as those in older women. Hyperemia of blood vessels and inflammatory infiltration, covered with urinary epithelium and squamous 
epithelium, may eventually form polyps (4). However, other histological types of polyps, such as inverted papilloma and fibroepithelial polyps, have also been reported (1).

Urethral polyp usually occurs in boys and seldom in young girls. In boys, polyps usually occur in the posterior urethra, usually close to the urethra membrane. They often present as a single tumor, rarely appearing as multiple tumors. In girls, the location of urethral polyps is nonspecific, and they can occur at the distal, middle or proximal urethra (4). The clinical manifestations of urethral polyp are intermittent, meaning there are intervals in which the condition is asymptomatic during onset. The location of pedicled polyps in the urethra changes with the flow of urine, which can lead to corresponding clinical symptoms. A more specific urodynamic model called "tower" uroflow rate curve exists in boys (2). Despite the difficulties in explaining this phenomenon accurately, the movement of polyps in the urethra stimulating the contraction and expansion of muscles in different regions of the urethra may be the reason for this urodynamic model. There is no such specific pattern in female patients. In some cases, urinary retention, dripping urine, interruption of urine flow, or hematuria can be experienced when the polyps are embedded in the external sphincter.

The diagnosis of urethral polyp should be based on a clinical triad, which in this case is defined as intermittent urine retention, intermittent hematuria, and lower urinary tract symptoms. Ultrasonography of the urinary system and micturition cystourethrography are of great significance in the diagnosis, while cystoscopy is the most important examination. The differential diagnosis of urethral polyps is: (I) changes in the histological structure of the urethra itself which may cause a certain degree of obstruction, including pathological changes in the navicular fossa (5), anterior urethral valve (6) or dilated Cowper (7); (II) polyps originating from the grape sarcoma in girls (2); and (III) mental urinary retention (2).

The conventional treatment of urethral polyps is transurethral resection (8). However, an open resection may be necessary when the polyp exceeds $3 \mathrm{~cm}$ in length and $1 \mathrm{~cm}$ in diameter, or it may move into the bladder. For small polyps in older children, foreign body forceps or stone baskets could be used to remove the tissues. For a large polyp, segmentation could initially be carried out, with the fragments then taken out of the urethra. However, this means the size of the polyp cannot be accurately measured. The operation should be conducted very gently and with great accuracy, to avoid iatrogenic urethral injury and secondary urethral obstruction. The recurrence can be avoided by burning the polyp's base after the polyp has been completely removed.

\section{Conclusions}

Urethral polyp should be considered first when a child experiences intermittent urine retention, intermittent hematuria, and intermittent lower urinary tract symptoms. It should also be considered as a possibility when there is a sudden interruption of urinary flow without obvious bladder or urinary calculus. The final specific diagnosis of polyps should be based on urethrocystoscope, and endoscopic resection should be the first choice of treatment.

\section{Acknowledgments}

Funding: This work was supported by the Science and Technology Innovation Plan of Jilin Provincial Science \& Technology Department, China (No. 20170204047SF).

\section{Footnote}

Conflicts of Interest: All authors have completed the ICMJE uniform disclosure form (available at http://dx.doi. org/10.21037/tp.2020.03.05). The authors have no conflicts of interest to declare.

Ethical Statement: The authors are accountable for all aspects of the work in ensuring that questions related to the accuracy or integrity of any part of the work are appropriately investigated and resolved. Written informed consent was obtained from the patient for publication of this manuscript and any accompanying images.

Open Access Statement: This is an Open Access article distributed in accordance with the Creative Commons Attribution-NonCommercial-NoDerivs 4.0 International License (CC BY-NC-ND 4.0), which permits the noncommercial replication and distribution of the article with the strict proviso that no changes or edits are made and the original work is properly cited (including links to both the formal publication through the relevant DOI and the license). See: https://creativecommons.org/licenses/by-nc-nd/4.0/

\section{References}

1. Kimura T, Miyazato M, Kawai S, et al. Urethral polyp in a 
young girl: a case report. Hinyokika Kiyo 2007; 53:657-9.

2. Akbarzadeh A, Khorramirouz R, Kajbafzadeh AM.

Congenital urethral polyps in children: report of

18 patients and review of literature. J Pediatr Surg 2014;49:835-9.

3. Beluffi G, Berton F, Gola G, et.al. Urethral polyp in a 1-month-old child. Pediatr Radiol 2005;35:691-3.

4. Ben-Meir D, Yin M, Chow CW, et al. Urethral polyps in prepubertal girls. J Urol 2005;174:1443-4.

5. Kajbafzadeh AM, Elmi A, Payabvash S, et al. The urethral valve of Guérin and lacuna magna: clinical presentations

Cite this article as: Du L, Zhu Y, Li Y, Sun Y. Ultrasonic manifestation of urethral polyp in a girl: a case report. Transl Pediatr 2020;9(3):262-265. doi: 10.21037/tp.2020.03.05 and urodynamic findings. Pediatr Surg Int 2007;23:873-8.

6. Kajbafzadeh AM, Payabvash S, Karimian G. Urodynamic changes in patients with anterior urethral valves: before and after endoscopic valve ablation. J Pediatr Urol 2007;3:295-300.

7. Satish KK, Mammen A, Ratani AA, Varma KK. Posterior urethral valves and Cowper's syringocele: A rare association causing voiding dysfunction. J Indian Assoc Pediatr Surg 2012;17:126-7.

8. Gleason PE, Kramer SA. Genitourinary polyps in children. Urology 1994;44:106-9. 